\title{
Lower bounds for blow up time of the $p$-Laplacian equation with damping term
}

\author{
Yavuz Dinç, Erhan Pişkin, Cemíl TunÇ
}

\begin{abstract}
In this work deals with the $p$-Laplacian wave equation with damping terms in a bounded domain. Under suitable conditions, we obtain a lower bounds for the blow up time. Our result extends the recent results obtained by Baghaei (2017) and Zhou (2015), for $p>2$.
\end{abstract}

\section{INTRODUCTION}

In this work, we study the following $p$-Laplacian equation with strong and weak damping terms

(1)

$$
\left\{\begin{array}{cl}
u_{t t}-\operatorname{div}\left(|\nabla u|^{p-2} \nabla u\right)-a \Delta u_{t}+b u_{t}=|u|^{q-2} u, \quad x \in \Omega, & t>0, \\
u(x, 0)=u_{0}(x), \quad u_{t}(x, 0)=u_{1}(x), & x \in \Omega, \\
u(x, t)=0, & x \in \partial \Omega, t>0,
\end{array}\right.
$$

where $\Omega \subset R^{n}(n=2,3, \ldots)$ is a bounded domain with a smooth boundary $\partial \Omega$; and $u_{0}(x) \in W_{0}^{1, p}(\Omega), u_{1}(x) \in L^{2}(\Omega), p>2 . a \geq 0, b>-a \rho_{1}$ with $\rho_{1}>0$ is the first eigenvalue of the operator $-\Delta$ under homogeneous Dirichlet boundary conditions and

$$
\left\{\begin{array}{l}
2<q<\infty \text { if } n=2, \\
2<q \leq\left\{\begin{array}{ll}
\frac{2 n}{n-2}, & \text { for } a>0 \\
\frac{2 n-2}{n-2}, & \text { for } a=0
\end{array} \text { if } n \geq 3 .\right.
\end{array}\right.
$$

When $p=2,(1)$ is reduced to the following wave equation

$$
u_{t t}-\Delta u-a \Delta u_{t}+b u_{t}=|u|^{q-2} u .
$$

In 2006, Gazzalo and Squassina [2] studied problem (3). They proved the local existence, global existence and blow up of solutions. Later, some authors studied the lower bounds for the blow up time under some conditions,

2020 Mathematics Subject Classification. Primary: 40B05; Secondary: 33E99.

Key words and phrases. Lower bounds, $p$-Laplacian equation, Damping term.

Full paper. Received 8 October 2020, accepted 30 March 2021, available online 15 June 2021.

*The authors are grateful to DUBAP (ZGEF.18.002) for research funds. 
see $[1,7,9]$. Also, in recent years, some authors investigate the lower bounds for blow up time for hyperbolic type equations, see $[3,4,6,8]$.

Inspired by the above papers, in this paper we consider the lower bound for the blow up time of solutions (1). Our result improves the recent results obtained by Baghaei [1] and Zhou [9], for $p>2$.

Now, for the problem (1), we define the functionals, the potential well depth $d$ and the unstable set $U$, are given as

$$
\begin{aligned}
I(u) & =\|\nabla u\|_{p}^{p}-\|u\|_{q}^{q}, \\
J(u) & =\frac{1}{p}\|\nabla u\|_{p}^{p}-\frac{1}{q}\|u\|_{q}^{q}, \\
E(t) & :=E\left(u, u_{t}\right)=J(u)+\frac{1}{2}\left\|u_{t}\right\|^{2}, \\
d & =\inf _{u \in H_{0}^{1}(\Omega) /\{0\} \quad \max J(\lambda u),}^{\lambda \geq 0} \\
U & =\left\{u \in W_{0}^{1, p}: J(u) \leq d \text { and } I(u)<0\right\}
\end{aligned}
$$

with above assumptions, the results on the existence of local solutions and the nonexistence of the solutions of the problem (1) can be reformulated as follows:

(i) Assume that $q$ satisfy (2), then there exist $T>0$ and a unique solution $u$ problem (1) satisfying

$$
\begin{aligned}
u & \in C\left([0, T), W_{0}^{1, p}(\Omega)\right) \cap C^{1}\left([0, T), L^{2}(\Omega)\right) \cap C^{2}\left([0, T), H^{-1}(\Omega)\right), \\
u_{t} & \in L^{2}\left((0, T), H_{0}^{1}(\Omega)\right) .
\end{aligned}
$$

(ii) The solution $\mathrm{u}$ of problem (1) blows up at a finite time $T$ if and only if there exists $\bar{t} \in[0, T)$ such that $u(\bar{t}) \in U$ and $E\left(u(\bar{t}), u_{t}(\bar{t})\right) \leq d$.

$$
\begin{aligned}
& E(t)=\frac{1}{2}\left\|u_{t}\right\|^{2}+\frac{1}{p}\|\nabla u\|_{p}^{p}-\frac{1}{q}\|u\|_{q}^{q}, \\
& E(0)=\frac{1}{2}\left\|u_{1}\right\|^{2}+\frac{1}{p}\left\|\nabla u_{0}\right\|_{p}^{p}-\frac{1}{q}\left\|u_{0}\right\|_{q}^{q} .
\end{aligned}
$$

Lemma 1 ([5]). Let $\Omega \subset R^{n}(n \geq 2)$. Let $u$ be a non-negative piecewise $C^{1}$ function defined in $\Omega$ with $u(x)=0, x \in \partial \Omega$. So

$$
\int_{\Omega}|u|^{2 s} \mathrm{~d} s \leq \delta\left(\int_{\Omega}|\nabla u|^{2} \mathrm{~d} x\right)^{s}
$$

satisfies for

$$
\left\{\begin{array}{cl}
s>1, & \text { if } n=2, \\
1<s<\frac{n}{n-2}, & \text { if } n \geq 3,
\end{array}\right.
$$

with $\delta=\left(\frac{n-1}{n^{\frac{3}{2}}}\right)^{2 s}|\Omega|^{1-\frac{(n-2)}{n} s}$. 
Theorem 1. Assume that $q>\frac{p+2}{2}$ holds. Let $u(x, t)$ be the solution of the problem (1), which blows up at a finite time $T^{*}$. Then

$\int_{\psi(0)}^{\infty} \frac{\mathrm{d} \tau}{q M+\tau+\varepsilon q p^{\frac{2(q-1)}{p}} 2^{\frac{2(q-1)}{p}-2} M^{\frac{2(q-1)}{p}}+\varepsilon p^{\frac{2(q-1)}{p}} q^{\frac{p-2 q+2}{p}} 2^{\frac{2(q-1)}{p}-2} \tau^{\frac{2(q-1)}{p}}} \leq T^{*}$, where

$$
\left\{\begin{array}{l}
\psi(0)=\left\|u_{0}\right\|_{q}^{q}, \\
\varepsilon=\left(\frac{n-1}{n^{\frac{3}{2}}}\right)^{2(q-1)}|\Omega|^{1-\frac{(n-2)(q-1)}{n}}, \\
M=\frac{1}{2}\left\|u_{1}\right\|^{2}+\frac{1}{p}\left\|\nabla u_{0}\right\|_{p}^{p}-\frac{1}{q}\left\|u_{0}\right\|_{q}^{q} .
\end{array}\right.
$$

Proof. Multiplying the equation of (1) by $u_{t}$, then integrating the result over $\Omega$, we have

$$
\begin{aligned}
& \int_{\Omega} u_{t}\left[u_{t t}-\operatorname{div}\left(|\nabla u|^{p-2} \nabla u\right)-a \Delta u_{t}+b u_{t}\right] \mathrm{d} x=\int_{\Omega} u_{t}|u|^{q-2} u \mathrm{~d} x, \\
& \frac{1}{2} \frac{\mathrm{d}}{\mathrm{d} t} \int_{\Omega}\left|u_{t}\right|^{2} \mathrm{~d} x+\frac{1}{p} \frac{\mathrm{d}}{\mathrm{d} t} \int_{\Omega}|\nabla u|^{p} \mathrm{~d} x+a \int_{\Omega}\left|\nabla u_{t}\right|^{2} \mathrm{~d} x+b \int_{\Omega}\left|u_{t}\right|^{2} \mathrm{~d} x \\
& =\frac{1}{q} \frac{\mathrm{d}}{\mathrm{d} t} \int_{\Omega}|u|^{q} \mathrm{~d} x .
\end{aligned}
$$

Since

$$
\frac{\mathrm{d}}{\mathrm{d} t}\left(\frac{1}{2}\left\|u_{t}\right\|^{2}+\frac{1}{p}\|\nabla u\|_{p}^{p}-\frac{1}{q}\|u\|_{q}^{q}\right)=-a\left\|\nabla u_{t}\right\|^{2}-b\left\|u_{t}\right\|^{2},
$$

the above calculations imply $E^{\prime}(t) \leq 0$, with

$$
E(t):=\frac{1}{2}\left\|u_{t}\right\|^{2}+\frac{1}{p}\|\nabla u\|_{p}^{p}-\frac{1}{q}\|u\|_{q}^{q} .
$$

So,

$$
E(t) \leq E(0)=M
$$

where $M$ is defined in (7) and

$$
\left\|u_{t}\right\|^{2}+\frac{2}{p}\|\nabla u\|_{p}^{p}=2 E(t)+\frac{2}{q}\|u\|_{q}^{q} \leq 2 M+\frac{2}{q}\|u\|_{q}^{q} .
$$

Now define

$$
\psi(t)=\|u\|_{q}^{q},
$$

then thanks to Cauchy's and embedding $\left(L^{p}(\Omega) \hookrightarrow L^{2}(\Omega), p>2\right)$ inequalities and Lemma 1 with $s=q-1$ and $\delta=\varepsilon$, we obtain

$$
\begin{aligned}
\psi^{\prime}(t) & =q \int_{\Omega}|u|^{q-2} u u_{t} \mathrm{~d} x \\
& \leq \frac{q}{2}\left(\left\|u_{t}\right\|^{2}+\|u\|_{2(q-1)}^{2(q-1)}\right) \\
& \leq \frac{q}{2}\left(\left\|u_{t}\right\|^{2}+\varepsilon\|\nabla u\|_{2}^{2(q-1)}\right)
\end{aligned}
$$




$$
\begin{aligned}
& \leq \frac{q}{2}\left(\left\|u_{t}\right\|^{2}+\varepsilon\|\nabla u\|_{p}^{2(q-1)}\right) \\
& =\frac{q}{2}\left(\left\|u_{t}\right\|^{2}+\varepsilon\left(\|\nabla u\|_{p}^{p}\right)^{\frac{2(q-1)}{p}}\right) .
\end{aligned}
$$

By the (9) and definition of $\psi$, we get

$$
\begin{aligned}
\psi^{\prime}(t) \leq & \frac{q}{2}\left[2 M+\frac{2}{q} \psi(t)+\varepsilon\left(\frac{p}{2}\right)^{\frac{2(q-1)}{p}}\left(2 M+\frac{2}{q} \psi(t)\right)^{\frac{2(q-1)}{p}}\right] \\
\leq & \frac{q}{2}\left[+\varepsilon\left(\frac{p}{2}\right)^{\frac{2(q-1)}{p}} 2^{\frac{2(q-1)}{p}-1}\left((2 M)^{\frac{2(q-1)}{p}}+\left(\frac{2}{q} \psi(t)\right)^{\frac{2(q-1)}{p}}\right)\right] \\
= & \left.\frac{q}{2}\left[2 M+\frac{2}{q} \psi(t)+\varepsilon^{\frac{p}{2}}\right)^{\frac{2(q-1)}{p}}\left((2 M)^{\frac{2(q-1)}{p}}+\left(\frac{2}{q} \psi(t)\right)^{\frac{2(q-1)}{p}}\right)\right] \\
= & q M+\psi(t)+\varepsilon q^{\frac{2(q-1)}{p}}\left(2^{\frac{2(q-1)}{p}} M^{\frac{2(q-1)}{p}}+2^{\frac{2(q-1)}{p}} q^{\frac{2(q-1)}{p}}(t)\right] \\
= & \left.q M+\psi(t)+\varepsilon q p^{\frac{2(q-1)}{p}} 2^{\frac{2(q-1)}{p}-2} M^{\frac{2(q-1)}{p}}\right) \\
& +\varepsilon p^{\frac{2(q-1)}{p}} q^{\frac{p-2 q+2}{p}} 2^{\frac{2(q-1)}{p}-2} \psi^{\frac{2(q-1)}{p}}(t),
\end{aligned}
$$

where used $(a+b)^{\varpi} \leq 2^{\varpi-1}\left(a^{\varpi}+b^{\varpi}\right)$. Thus, the above inequality implies that

$$
\begin{aligned}
\frac{\mathrm{d} \psi(t)}{\mathrm{d} t} \leq & q M+\psi(t)+\varepsilon q p^{\frac{2(q-1)}{p}} 2^{\frac{2(q-1)}{p}-2} M^{\frac{2(q-1)}{p}} \\
& +\varepsilon p^{\frac{2(q-1)}{p}} q^{\frac{p-2 q+2}{p}} 2^{\frac{2(q-1)}{p}-2} \psi^{\frac{2(q-1)}{p}}(t) .
\end{aligned}
$$

Since $\lim _{t \rightarrow T^{*}} \psi(t)=\infty$, we get from (10)

$\int_{\psi(0)}^{\infty} \frac{\mathrm{d} \tau}{q M+\tau+\varepsilon q p^{\frac{2(q-1)}{p}} 2^{\frac{2(q-1)}{p}-2} M^{\frac{2(q-1)}{p}}+\varepsilon p^{\frac{2(q-1)}{p}} q^{\frac{p-2 q+2}{p}} 2^{\frac{2(q-1)}{p}-2} \tau^{\frac{2(q-1)}{p}}} \leq T^{*}$.

This completes the proof of the main theorem.

\section{Conclusion}

In this work, we obtained the lower bounds for blow up time of the nonlinear $p$-Laplacian equation with damping terms in a bounded domain. This improves and extends many results in the literature. 


\section{REFERENCES}

[1] K. Baghaei, Lower bounds for the blow-up time in a superlinear hyperbolic equation with linear damping term, Computers and Mathematics with Applications, 73 (2017), 560-564.

[2] F. Gazzola, M. Squassina, Global solutions and finite time blow up for damped wave equations, Annales de l'Institut Henri Poincaré, Analyse Non Linéaire, 23 (2006), 185-207.

[3] X. Peng, Y. Shang, X. Zheng, Lower bounds for the blow-up time to a nonlinear viscoelastic wave equation with strong damping, Applied Mathematics Letters, 76 (2018), 66-73.

[4] A. Peyravi, F. Tahamtani, Upper and lower bounds of blow up time to a strongly damped wave equation of Kirchhoff type with memory term and nonlinear dissipations, Mediterranean Journal of Mathematics, 15 (2018), 1-16.

[5] G.A. Philippin, Lower bounds for blow-up time in a class of nonlinear wave equation, Zeitschrift für angewandte Mathematik und Physik, 66 (2015), 129-134.

[6] E. Pişkin, Y. Dinç, C. Tunç, Lower bounds for the blow up time to a coupled nonlinear hyperbolic type equations, Communications in Advanced Mathematical Sciences, 3 (1) (2020), 53-56.

[7] L. Sun, B. Guo, W. Gao, A lower bound for the blow-up time to a damped semilinear wave equation, Applied Mathematics Letters, 37 (2014), 22-25.

[8] S.T. Wu, Lower and upper bounds for the blow-up time of a class of damped fourthorder nonlinear evolution equations, Journal of Dynamical and Control Systems, 24 (2018), 287-295.

[9] J. Zhou, Lower bounds for blow-up time of two nonlinear wave equations, Applied Mathematics Letters, 45 (2015), 64-68.

Yavuz Ding

Department of Electronics

Technical Mardin Artuklu University

MARDiN

TURKEY

E-mail address: yavuzdinc@artuklu.edu.tr

\section{ERHAN PişKíN}

Department of Mathematics

Dícle UNIVERSITY

21280, DiYARbakir

TURKEY

E-mail address: episkin@dicle.edu.tr

\section{Cemil TunÇ}

Department of Mathematics

VAN YÜZÜNCÜ YIL UNIVERSITY

VAN

TURKEY

E-mail address: cemtunc@yahoo.com 